\title{
Rafter (Nicole), The Origins of Criminology. A Reader
}

Edited by Nicole Rafter, Oxon, New York, Routledge, 2009, 348 pp., avec quelques illustrations, ISBN 980415451123

\section{Michel Porret}

\section{(2) OpenEdition}

Édition électronique

URL : https://journals.openedition.org/chs/1209

DOI : 10.4000/chs.1209

ISSN : 1663-4837

Éditeur

Librairie Droz

\section{Édition imprimée}

Date de publication : 1 décembre 2010

Pagination : 142-144

ISBN : 978-2-600-01470-0

ISSN : 1422-0857

\section{Référence électronique}

Michel Porret, "Rafter (Nicole), The Origins of Criminology. A Reader ", Crime, Histoire \& Sociétés / Crime, History \& Societies [En ligne], Vol. 14, n² | 2010, mis en ligne le, consulté le 23 mars 2022. URL : http:// journals.openedition.org/chs/1209; DOI : https://doi.org/10.4000/chs.1209 
Rafter (Nicole), The Origins of Criminology. A Reader, Edited by Nicole Rafter, Oxon, New York, Routledge, 2009, 348 pp., avec quelques illustrations, ISBN 980415451123

À l'instar de la médecine légale, la criminologie est devenue la science auxiliaire de la justice pénale contemporaine. Ce livre en montre l'enracinement théorique.

7 Jean-Claude Farcy, Dominique Kalifa et Jean-Noël Luc (dir.), L'Enquête judiciaire en Europe au XIX $X^{e}$ siècle: acteurs, imaginaires, pratiques, Paris, Créaphis, 2007, 392 pp. Voir aussi le petit dossier «L'enquête judiciaire et ses récits », Revue d'histoire du XIX ${ }^{e}$ siècle, $2008, \mathrm{n}^{\circ} 36$. 
Conclue d'une substantielle bibliographie introductive à l'histoire des sciences du crime, cette petite et pratique anthologie est divisée en dix parties. Avec un zeste de téléologie, Nicole Rafter, traductrice en anglais de Lombroso, rassemble un corpus de textes criminologiques classiques, publiés principalement au XIX ${ }^{\mathrm{e}}$ siècle. Cette période est celle durant laquelle la «science du crime»s'autonomise et gagne ses lettres de noblesse avec son implantation institutionnelle, ses publications scientifiques, ses congrès, ses réseaux intellectuels, ses associations professionnelles, ses groupes de pression. S'y ajoute la formulation de sa doctrine qui est une sociologie analytique et normative de l'anomie sociale, fortement empreinte de morale. La courte introduction de l'auteur (pp. XIII-XXVI), qui commente ensuite en quelques paragraphes chacun des 61 textes sélectionnés, évoque l'enracinement urbain des sciences du crime au siècle de Marx. En insistant sur les «origines» toujours improbables d'un objet historique, Nicole Rafter voit notamment chez Beccaria (prévention légale du crime comme fait social) et chez Lavater (physiognomonie comme anthropologie morale soudant l'apparence physique au comportement) les «précurseurs » de la pensée criminologique. Celle-ci trouve un relais efficace et inspirateur chez Philippe Pinel et ses confrères aliénistes (James Cowles Prichard, Daniel Hack Tuke, Richard von Krafft-Ebing ou encore Prosper Despine). À leur suite, en amont et en aval de Darwin, les doctrinaires évolutionnistes de la dégénérescence et de l'atavisme héréditaire (Bénédict-Auguste Morel, J. Bruce Thompson, le prudent Henry Maudsly pour qui la frontière entre la folie et la santé mentale n'est pas établie) labourent intellectuellement le terreau des économistes et des penseurs du déclassement social comme condition du paupérisme industriel, du crime primaire ou commis en récidive (Elizabeth Fry, Joseph John Gurney, Friederich Engels, Edwards Craspey, etc.). Avant et après Cesare Lombroso, l'anthropologie physique et mentale de l'homme né ou devenu criminel en opère la taxinomie. Son cerveau prouvera ou infirmera la déchéance dans le crime ponctuel ou d'habitude (J. Bruce Thompson, Moriz Benedikt, Pauline Tarnowsky, Havelock Ellis, Arthur Mac Donald, Léonce Manouvrier, Alexandre Lacassagne, Frederik Howards). À la Belle Époque, pour notamment identifier les délinquants endurcis, la science du crime devient un savoir indiciaire. Elle recoupe les techniques signalétiques, dont le bertillonnage (photographie de «face et de profil») et la culture de l'empreinte digitale du spécialiste de l'hérédité Francis Galton. Parfois obsédé par la dégénérescence et les «sciences de l'hérédité» comme mesure de l'évolution physique et mentale, le savoir criminologique fraie avec l'eugénisme (Johann Gaspard Spurzheim, Josephine Shaw Lowell, Henry M. Boies, W. Duncan McKim) dans une finalité de prévention de l'anomie sociale. Les statistiques criminelles la mesurent de plus en plus systématiquement en ville (problème du «milieu») et dans les observatoires spécialisés que sont notamment les prisons (Andre-Michel Guerry, Adolphe Quetelet, Joseph Fletcher, Mary Carpenter, Frederick Howard Wines, Enrico Ferri). Finalement, la criminalité dans toutes ses formes est pensée comme une donnée de la vie sociale moderne (rapports entre hommes et femmes, industrialisation, ville, paupérisme et «classes dangereuses», etc.) que problématisent les économistes, les observateurs empiriques ou scientifiques comme Francis Lieber, Friederich Engels, Henry Mayhew, John Binny, Gabriel Tarde, Edward Alsworth Ross, Émile Durkheim, Enrico Ferri. Les textes choisis montrent qu'à l'instar des autres sciences sociales dont elle se dégage ou qu'elle inspire, la criminologie comme savoir «objectif» sur la délinquance évolue lentement de savoirs diffus à un savoir constitué. Elle s'émancipe en s'en inspirant de la médecine et de la psychiatrie légales. Elle ouvre la voie 


\section{4}

à la police scientifique selon Edmond Locard (Lyon) ou Rodolphe Archibald Reiss (Lausanne). Les experts du passage à l'acte criminel adhèrent au grand idéal positiviste de leur temps. Ceci les autorise à bricoler un savoir qui veut quantifier et modéliser pour le prévenir l'anomie sociale comme origine ou conséquence du crime. Tout autour de la dégénérescence darwinienne des espèces donc des individus déclassés, utilisant la prison comme un laboratoire pour évaluer la dangerosité de l'homo criminalis récidiviste ou non, les criminologues en pointent les causalités atavique ou sociale comme le font, partiellement, les savants d'obédience lombrosienne ou ceux qui suivent le Lyonnais Alexandre Lacassagne. La criminologie conforte le positivisme pénal dans la plupart des États régis par la légalité des délits et des peines. L'anthologie proposée par Nicole Rafter montre les enjeux descriptifs et normatifs de la pensée criminologique comme science «objective» et «exacte» (rêve s'il en est !) du comportement et de la personnalité de l'homo criminalis au fil du long $\mathrm{XIX}^{\mathrm{e}}$ siècle.

Michel Porret Université de Genève Michel.Porret@unige.ch 